\title{
Tsafon
}

Revue d'études juives du Nord

$76 \mid 2018$

Expressions yiddish de la nature

\section{Les mondes dépeuplés de Reïzl Zychlinsky}

\section{Fleur Kuhn-Kennedy}

\section{OpenEdition}

Journals

Édition électronique

URL : https://journals.openedition.org/tsafon/1310

DOI : $10.4000 /$ tsafon. 1310

ISSN : 2609-6420

\section{Éditeur}

Association Jean-Marie Delmaire

\section{Édition imprimée}

Date de publication : 1 décembre 2018

Pagination : $97-112$

ISSN : $1149-6630$

\section{Référence électronique}

Fleur Kuhn-Kennedy, «Les mondes dépeuplés de Reïzl Zychlinsky », Tsafon [En ligne], 76 | 2018, mis en ligne le 30 juin 2019, consulté le 25 juin 2021. URL : http://journals.openedition.org/tsafon/1310 ; DOI : https://doi.org/10.4000/tsafon.1310 


\title{
Les mondes dépeuplés de Reïzl Zychlinsky
}

\author{
Fleur Kuhn-Kennedy ${ }^{*}$
}

«Celui qui, en voyageant, médite la Loi et interrompt sa méditation pour s'écrier : Que cet arbre est beau ! Que ce champ est bien cultivé ! Celui-là, selon l'Écriture, compromet sa vie ${ }^{1}$. Cette maxime, qui figure dans un traité bien connu de la Mishna, était familière à tous les hommes juifs d'Europe orientale rompus dès leur plus jeune âge à la connaissance de la tradition rabbinique. C'est dire que délaisser la médiation du système de valeurs culturel pour retrouver, à la faveur de l'instant, une forme de communion avec le naturel, constituait, selon le code éthique de ces sociétés, une incartade frappée de soupçon, voire une menace pour l'intégrité humaine, dont l'existence reposait sur la distinction du corps, individuel ou collectif, avec l'environnement dans lequel il évoluait. Non que la nature soit absente des textes fondateurs et de ceux qu'ils ont inspirés: il suffit de lire les premières pages de la Genèse pour comprendre l'importance de la terre, de l'eau, de la faune et de la flore en tant que constituants du monde de Dieu. À lire la littérature yiddish moderne, on trouve mille traces de la manière dont les paysages bibliques innervaient l'imaginaire des enfants soumis dès leur plus jeune âge aux contraintes de l'apprentissage des textes et de la Loi. Pourtant, même dans ce qui peut apparaitre comme des escapades coupables hors de l'écriture, la relation entre l'homme et la nature passe par la médiation du texte, qui réaffirme la singularité de l'Homme en tant que celui qui nomme et qui met en signes. Qu'on pense par exemple au petit Yekhiel,

\footnotetext{
${ }^{*}$ Centre d'Études et de Recherches Comparatistes, Sorbonne Nouvelle - Paris 3.

${ }^{1}$ Pirkei Avot [Maximes des Pères] 3, 9. Pour l'édition française, voir Les Maximes des pères, trad. Moïse Schuhl, Paris, Colbo,1986.
} 
héros du Juif aux psaumes ${ }^{2}$ qui, dans ce roman de Sholem Asch, regarde la Vistule et croit voir le Jourdain, scrute les collines de Pologne et imagine le mont Ararat. On ne saurait douter de la symbiose qui, pour ce personnage, unit le $v u$ et le $s u$, le monde matériel et les outils symboliques qui l'informent. Yekhiel ne fait pas l'expérience directe de la nature; il ne la voit que par la médiation dont ses lectures chargent le paysage, aux dépens de toute vraisemblance géographique et historique. Toujours est-il que le jeune garçon, justement parce qu'il n'arrive pas à dépasser l'enchantement du narratif et du descriptif pour entrer dans des raisonnements plus abstraits, devient un «juif aux Psaumes », c'est-àdire un ignorant, tout juste bon à réciter des louanges, dans un rapport purement affectif au divin qui, s'il est acceptable pour les femmes, dont le rôle réside avant tout dans la préservation de la pureté domestique, est indigne des hommes, supposés consacrer leur vie à l'étude et à l'interprétation des textes sacrés. C'est pourquoi, jusqu'au XIX ${ }^{\mathrm{e}}$ siècle qui voit l'émergence du hassidisme, puis la modernisation des sociétés juives, la sensibilité pour la nature semble inextricablement liée au féminin, au même titre que la lecture du yiddish, langue prétendument dévolue à des sujets moins élevés que l'hébreu et l'araméen des textes fondateurs. Non que les femmes soient les seules à lire ou à écrire en yiddish - au contraire, si le public visé est féminin, les auteurs sont souvent des hommes, et la pratique du vernaculaire est courante dans toutes les sphères de la vie juive, y compris celles qui concernent la discussion de questions exégétiques pointues -, mais les valeurs associées à la langue s'accordent à la distribution genrée des rôles sociaux, érigeant l'hébreu en émissaire d'un discours érudit, intellectuel et moral tandis que le yiddish se trouve relégué à la sphère du quotidien, de l'immédiat, des émotions, à l'expression du monde matériel et sensible.

Sans doute n'est-ce donc pas un hasard si, en même temps que de nouveaux courants de pensée réhabilitent le yiddish - d'abord comme langue du chant, de la prière, du conte, de la communication avec Dieu, puis comme langue d'une culture séculière attachée à des idéaux diasporistes et socialistes, et enfin comme langue de création d'une littérature d'avant-garde occupée à exprimer une nouvelle vision du monde et du verbe - l'attention à la nature s'amplifie, acquérant de nouvelles significations à mesure que se fragmente l'expérience juive et

\footnotetext{
${ }^{2}$ Sholem Asch, Le Juif aux psaumes, trad. Juliette Pary et Isaac Pougatch, Paris, Flammarion, 1971, p. 17-18.
} 
que se déplacent les espaces, géographiques ou symboliques, dans lesquels elle se forme. On voit alors se dessiner une vision résolument moderne de l'interaction entre l'homme et le monde, alimentée par le romantisme, le naturalisme et l'histoire littéraire européenne, reconfigurée par les parcours migratoires qui exposent les Juifs d'Europe de l'Est à de nouveaux paysages, recomposée par l'effritement de l'ordre divin. Au sein de cette modernité, les écrivains femmes occupent une place notable, quoique moins visible que celle des grands noms masculins de la littérature de la fin du $\mathrm{XIX}^{\mathrm{e}}$ et du $\mathrm{XX}^{\mathrm{e}}$ siècle. S'illustrant souvent dans le genre poétique, à la fois pour des raisons matérielles et parce que, comme le note Irena Klepfisz, elles sont plus facilement acceptées dans ce domaine, «la prose [étant] souvent associée aux questions intellectuelles, la poésie aux sentiments ${ }^{3}$, elles ont participé à toutes les transformations politiques et esthétiques du $\mathrm{XX}^{\mathrm{e}}$ siècle tout en demeurant pour beaucoup d'entre elles en retrait des mouvements et des écoles de pensée dans lesquels se réunissaient leurs contemporains.

Parmi elles, un nom mérite une attention particulière : celui de Reïzl Zychlinsky (1910-2001), poétesse dont la vie et l'œuvre ont traversé une bonne partie du siècle et recouvrent une trajectoire qui l'a menée de Pologne en Union soviétique, puis, après un bref retour dans son pays natal au lendemain de la guerre, à Paris, où elle est restée trois ans avant d'émigrer aux États-Unis. Très tôt saluée par le mouvement introspectionniste américain, dont elle reçoit le prix Reuben Ludwig en 1937 à la suite de la parution de son premier recueil, et en particulier par Yankev Glatstein, qui reconnaît dans son écriture une parenté, selon lui surprenante chez une "jeune fille de Gombin » ${ }^{4}$, avec le modernisme yiddish américain, elle a publié en tout sept recueils dont la parution s'échelonne de 1936 à $1993^{5}$ et qui, dans une attention exacerbée aux

\footnotetext{
${ }^{3}$ Irena Klepfisz, «Queens of Contradiction: A Feminist Introduction to Yiddish Women Writers », introduction à : Frieda Forman et al. (éd.), Found Treasures. Stories by Yiddish Women Writers, Toronto, Second Story Press, 1994, p. 48-49 : «Prose tends to be associated with intellectual matters, poetry with feeling ».

${ }^{4}$ Yankev Glatshteyn, « Reyzl Zychlinsky », dans In tokh genumen, New York, Farband, 1956, p. 336 : « gombiner meydl».

${ }^{5}$ Lider [Poèmes], 1936 ; Der regn zingt [La pluie chante], 1939; Tsu loytere bregn [Vers de clairs rivages], 1948 ; Shvaygndike tirn [Portes muettes], 1962 ; Harbstike skvern [Squares d'automne], 1969 ; Di november-zun [Le soleil de novembre], 1977 ; Naye lider [Nouveaux poèmes], 1993. De ces recueils, seul le quatrième a été traduit en français (Portes muettes, trad. et préface Rachel Ertel, Paris, L'improviste, 2007). Des poèmes sont également disponibles en anglais dans des anthologies de poésie, ainsi que dans le recueil de poèmes choisis God Hid His Face, Santa Rosa, Word \& Quill Press, 1997. Voir en ligne : http://www.ibiblio.org/yiddish/Book/Zychlinsky/rz-cont.html
} 
objets, aux paysages, aux lieux, inventent une écriture souvent dépeuplée de toute présence humaine manifeste, où la nature occupe une fonction quasi animiste. Cette écriture, certes, évolue en forme et en sens au fil des expériences de l'auteur, mais les mêmes motifs courent d'un recueil à l'autre, investissant les lieux natals et la réalité cosmique de valeurs différentes selon que le Je poétique est assumé par une jeune fille aspirant à de nouveaux horizons, par une femme ayant quitté sa bourgade pour la capitale, par une exilée arrivée en Union soviétique pour fuir les persécutions nazies ou par une émigrée échouée de l'autre côté de l'Atlantique après la disparition des siens. La vision littéraire par laquelle sa poésie informe le monde, tout en étant résolument personnelle et singulière, et dépourvue de toute appartenance idéologique revendiquée, participe des révolutions esthétiques et politiques qui animent le monde yiddish moderne.

\section{Terre et mère}

Lorsque Reïzl Zychlinsky publie son premier recueil de poèmes en 1936, ce dernier est assorti d'une préface d'Itzik Manger qui s'ouvre sur ces mots : "Après le recueil classique de Miriam Ulinover Le Trésor de ma grand-mère ${ }^{6}$, les poèmes de Reïzl Zychlinsky constituent une nouvelle vraie découverte de la sensibilité poétique féminine dans le monde juif de la Pologne du Congrès » ${ }^{7}$. D'emblée, le ton est donné : c'est en tant que femme, et en tant que porteuse d'une «sensibilité poétique féminine $»^{8}$ que la poétesse se trouve évaluée. La dimension féminine de l'écriture ainsi que de la position énonciative ne constitue pas un élément d'approche parmi d'autres; elle est conçue comme une caractéristique formant un champ en soi, au point que la comparaison avec d'autres œuvres plus anciennes ne semble pouvoir se faire qu'en regard des productions d'autres femmes écrivains. Après avoir évoqué les images d'arbres et d'oiseaux qui, dans le tout premier poème de

\footnotetext{
${ }^{6}$ Miryam Ulinover, Der bobes oytser, Varsovie, Haakhim Levin Epstein un shutfim, 1922. Il existe également une traduction française : Miryam Ulinover, Un bonjour du pays natal, édition et introduction de Natalia Krynicka, trad. Batia Baum, Paris, Bibliothèque Medem, 2003.

${ }^{7}$ Itzik Manger, «Forvort», dans Reïzl Zychlinsky, Lider, Varsovie, Biblyotek fun yidish PEN-klub, 1936, p. 3 : «Nokh Miryam Ulinovers klasishn lider bukh Der bobes oytser, zenen di lider fun Reyzl Zychlinsky a naye emese antplekung fun froyishpoetishn gemit in yidishn kroyn-poyln ».

${ }^{8}$ « froyish-poetish gemit ». Itzik Manger, « Forvort», dans Reïzl Zychlinsky, ibid., p. 3. Sauf mention contraire, les traductions sont de moi.
} 
l'auteure, suggéraient les sensations de l'automne, le préfacier poursuit en citant un second poème construit comme une adresse de l'enfant à sa mère, deux figures selon lui «centrales du lyrisme authentiquement féminin de Reïzl Zychlinsky ${ }^{9}$. On peut évidemment prendre le contrepied de cette position, qui se répète jusqu'à devenir lassante dans plusieurs articles consacrés à la poétesse ${ }^{10}$. Ces discours, faisant fi d'une indépendance qui la tient à l'écart de toute revendication collective, sont immédiatement caducs dès lors qu'ils postulent que sa voix propre ne peut être que le porte-parole de celle des femmes juives dans leur ensemble. Pourtant, si les lectures essentialisantes de l'écriture féminine demandent indéniablement à être contestées, il convient également de ne pas négliger ce que peut signifier, dans la poésie de Zychlinsky, le fait d'écrire, sinon au nom des femmes, du moins en tant que femme, et la manière dont cette position au sein de la société juive traditionnelle, puis $\mathrm{du}$ monde littéraire yiddish, travaille les formes et les thèmes dans lesquels elle s'illustre.

La terre, chez elle, n'est jamais patrie : elle est toujours féminine, lieu d'un ancrage maternel qui prend autant sa source dans l'histoire personnelle de l'auteure que dans la spécificité des ressources symboliques par lesquelles la culture juive donne sens au monde. En effet, selon la distribution des rôles sociaux favorisée par les structures juives communautaires, la place occupée par les hommes correspond à un espace symbolique, incarné par les livres sacrés : à travers leur étude, le père connecte sa famille à des générations d'exégètes et l'insère dans une chaîne de transmission temporelle; la place de la mère, en revanche, est plutôt liée à l'espace matériel, habité, au foyer dont elle a la charge de préserver la pureté : c'est elle la garante de l'ancrage terrestre. Par ailleurs, on sait que pour Reïzl Zychlinsky en particulier, l'image du père est synonyme d'errance : celui-ci, après avoir tenté d'émigrer par trois fois aux États-Unis, y est décédé en 1928, hors-lieu, hors ancrage familial, et comme désamarré de toute attache ${ }^{11}$. L'enfance de la poétesse

\footnotetext{
${ }^{9}$ Ibid., p. 4 : « di tsentral-figurn fun Reïzl Zychlinskys ekht froyisher lirik ».

${ }^{10}$ Chez Glatstein, elle devient même d'un paternalisme outrancier: celui-ci loue l'aptitude de la poétesse à « ne pas franchir la frontière qui la sépare de l'intelligence masculine » et remarque qu'elle affiche " quand il le faut une coquette niaiserie, qui tient en fait de la stratégie féminine ». À la lire, ajoute-t-il, " on peut entendre même les yeux fermés la parole d'une femme ». Cf. In tokh genumen, op. cit., p. 335.

${ }^{11}$ Voir à ce propos un poème du tout dernier recueil de Reïzl Zychlinski, « Mayn tates briv fun Amerike »: "Un efsher zol ikh forn keyn Shikago / Nokh an entfer? / Oyszukhn dort dos bes-oylem / Vu mayn tate rut in a keyver, / Un hot a matseyve tsu kopns, / A shteyn - » [Et peut-être que je devrais aller à Chicago / Chercher une
} 
est donc rythmée par ces absences, que viennent pallier le saule, les peupliers, le chat, la rivière, la cruche bleue, autant d'éléments qui, comme les visions saillantes d'un haïku, viennent ponctuer l'ensemble de son œuvre, indiquant le lieu et les saisons, créant un sentiment de pérennité intérieure lié à un univers résolument maternel.

Tout ce qui appartient au monde immuable du shtetl - d'ailleurs plutôt caractérisé par son paysage que par les interactions et les institutions qui en structurent la vie collective - est étroitement imbriqué dans un espace à la fois intime et absolu qui, dans les toutes premières expériences poétiques en tout cas, est investi de la même affectivité ambivalente que la figure de la mère: lieu de sécurité, de stabilité, d'enveloppement, il est aussi signe d'un enfermement qui crée l'aspiration à un ailleurs, vers la poursuite rêvée du lointain soleil et du vent voyageur. Il y a d'abord les poèmes dans lesquels la mère est directement évoquée, comme par exemple «Mame» [Maman], où la poétesse l'invite à bercer le vent dans son tablier pour l'endormir, ou «Dos alte lid» [La vieille chanson], où la course du soleil, étirant l'attente de quelque chose qui n'arrive pas, se met à déformer les corps, rendant étrangement inquiétantes et presque fantastiques les mains maternelles occupées aux tâches quotidiennes :

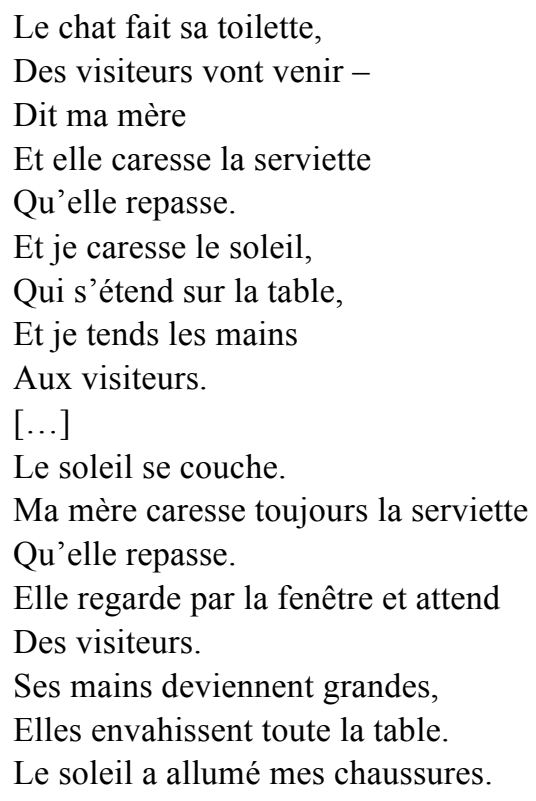

réponse ? / Aller en quête du cimetière / Où mon père repose dans un cercueil, / Avec à son chevet pour toute pierre tombale, / Une pierre -]. Cf. Naye lider, Tel Aviv, Yisroelbukh, 1993, p. 22. 
Je me lève.

Je vais à la rencontre de la vieille armoire :

La vieille armoire s'approche en boitant

Criblée de trous de vers à bois

Et de pluie. ${ }^{12}$

Plus encore que la mère et que la persona poétique, c'est ce qui frémit autour d'elles, la manière dont les objets se meuvent sous leur regard, qui est au centre du poème. Dans le déclin du soleil, la danse des ombres, le mouvement de l'armoire vermoulue, se disent à la fois l'attente toujours déçue du mari et du père - ce visiteur mystérieux qui jamais n'arrive -, la dimension sclérosante de l'espace familial et familier et l'aspiration à quelque chose d'innommé, au monde inconnu de l'avenir incarné par les chaussures, instruments du départ, et par le soleil, source de lumière et de chaleur venue d'un autre monde. C'est à travers ces objets que les sujets semblent sentir, se mouvoir et s'émouvoir. Il n'est ainsi pas rare que les protagonistes humains passent au second plan, voire qu'ils deviennent totalement absents, comme dans " A kind iz krank» [Un enfant est malade], où l'enfant tout indéfini et hypothétique du titre et du premier vers est livré aux bons soins d'une nature maternante, alors que la mère elle-même ne transparaît plus qu'en creux sous ses relais métaphoriques :

Un enfant est malade.

Les fleurs baissent la tête,

Coupables.

Le peuplier a enlacé de ses bras

Le toit de chaume

Et ne lâche pas de ses vieux yeux

Le chemin.

À l'aube un mendiant est passé par là,

Transperçant le brouillard de ses yeux bleus.

Et peut-être l'arbre attend-il la première étoile ?

La poule couveuse a pris sous ses ailes

Les quinze petits poussins jaunes

Et a fait tomber la nuit tôt.

12 « Di kats vasht zikh / S'veln kumen gest - / Zogt di mame / Un zi glet dos hantukh, / Vos zi prest. / Un ikh glet di zun, / Vos tsit zikh oyfn tish, / Un ikh tsi di hent oys, / Tsu di gest. / [...] / Di zun fargeyt. / Di mame glet nokh alts dos hantukh, / Vos zi prest. / Zi kukt in fenster un vart / Oyf gest. / Ire hent vern groys, / Farnemen shoyn dem gantsn tish. / Di zun hot ongetsundn mayne shikh. / Ikh hoyb zikh uf. / Ikh gey der alter shank antkegn : / Di alte shank kumt tsu hinken / Mit azoyfil lekher fun holtsverem / Un fun regn ». Voir Reïzl Zychlinsky, « Dos alte lid », Lider, op. cit., p. 10-11. 
La chatte, blottie en boule dans un coin,

Ronronne, ronronne,

Et le tabouret à trois pieds

Tend l'oreille en rêvant. ${ }^{13}$

Il n'est ici pas d'autre présence humaine que celle du mendiant, déréalisé par sa fonction purement symbolique, mais il est évident que l'image maternelle transpire dans tout ce qu'évoque le poème : la poule recueillant ses poussins sous son aile, le peuplier (nom féminin en yiddish) enlaçant le toit de la maison et, au-delà, tous les objets anthropomorphisés qui, parce qu'ils appartiennent au monde de la mère, à la terre et au foyer qui lient la poétesse à celle-ci, suffisent à suggérer sa présence.

Dans Tsu loytere bregn [Vers de clairs rivages], le recueil regroupant des poèmes écrits pendant la guerre et très tôt après la libération, au moment où Zychlinsky revient en Pologne après son exil en Union soviétique, l'image de la nature, des lieux de l'enfance et de la figure maternelle se modifient, non seulement parce que l'exil puis la destruction $\mathrm{du}$ foyer natal en font le lieu d'une origine devenue inaccessible plutôt que d'un enfermement subi, mais aussi parce qu'un nouveau lieu d'appartenance se met en place, au moins transitoirement, dans la Russie où Reïzl Zychlinsky est elle-même devenue mère. Le fils, dans les jeux duquel miroite tout un microcosme - le temps qui « frisotte en joyeuse écume ${ }^{14}$, les mains faisant «gicler l'eau en mille petits soleils $\gg^{15}-$, donne vie à un espace qui reconfigure le sentiment d'infini et de permanence en fonction de ce nouveau lien de filiation. Quant à la mère et aux paysages qui lui sont liés, s'ils sont toujours présents en tant qu'objets de manque, de douleur et de culpabilité, ils sont aussi relayés par d'autres figures maternelles et d'autres paysages, ancrés dans une vision soviétique où toute écriture devient idéologique. On a donc d'un côté un paysage devenu purement mémoriel où rien ne bougera jamais plus, ni la rivière, ni les saules, ni la cruche bleue, et qui semble voué à

\footnotetext{
${ }^{13}$ Ibid., p. 13 : « A kind iz krank. / Boygn blumen di kep arop / Shuldik. / Di topolye hot arumgenumen mit di hent / Dem shtroyenem dakh / Un kukt zikh oys di alte oygn / Oyfn veg. / S'iz fartog do adurkhgegangen a betler / Un durkhgeshnitn di neplekh mit bloye oygn. / Un efsher vart zi oyfn ershtn shtern ? / Di kvoke hot aruntergenumen unter di fliglekh / Di fuftsn gele hindelekh / Un fri gemakht nakht. / Di kats ligt ayngenuret in a knoyl in vinkl / Un mrutshet, mrutshet, / Un dos benkl oyf dray fis / Horkht un kholemt ».

14 «Kroyzlt zikh di tsayt / Mit a freylekhn shoym. » Reïzl Zychlinksy, « Mayn kind », Tsu loytere bregn, Lodz, Yidish-bukh, 1948, p. 33.

${ }^{15}$ Ibid., « Un tseshpritsn di vaser / Oyf toyznt kleyne zunen ».
} 
répéter indéfiniment quelques souvenirs sur le mode de la revenance, et de l'autre une posture poétique qui semble aux antipodes de celle qu'adoptait Zychlinsky dans ses premières productions, et de celle à laquelle elle reviendra par la suite. Le poème "Mames" [Mères] témoigne de ce tribut payé à l'esthétique soviétique, évoquant la terre de Russie à travers les femmes qui sacrifient leurs fils à la liberté, et suggérant en filigrane la dimension maternelle de la terre elle-même :

Les nuages sont trempés de sang,

La nuit tombe sur les champs de bataille -

J'ai quitté ma maison,

Quitté - sans fermer la porte.

Les nuages sont des mères grisonnantes :

Les cheveux ébouriffés, les mains tordues,

Ils se penchent sur les fils tués -

Les fils aux yeux crevés et arrachés.

Les nuages charrient dans les cieux

Depuis des milliers d'années leur chagrin de mère.

Les champs de bataille se couvrent de verdure

Et se gorgent de sang à nouveau.

La terre deviendra pierre,

Raide de sang et rassasiée de cris.

Les rivières se dessécheront

Tout comme les saules argentés qui les surplombent !

Tandis que tombent les bombes sur des villes endormies

Ruinant les maisons et tuant les hommes,

Une femme enceinte traverse le champ

Et prie Dieu qu'il lui fasse la grâce d'un fils. ${ }^{16}$

Comme l'a remarqué Karina von Tippelskirch ${ }^{17}$ dans sa monographie consacrée à la poétesse, la structure de ces poèmes engagés,

\footnotetext{
${ }^{16}$ Ibid., p. 29 : « Volkns zenen ayngetunken in blut, / Iber di shlakht-felder vert nakht / Ikh bin avek fun mayn heym, / Avek - un di tir nisht farmakht. // Di volkns zenen groye mames: / Di hor tseshoybert, di hent farbrokhn;/ Zey beygn zikh ayn iber derhargete zin - / Zenen di oygn bay di zin oysgeshtokhn. // Shvimen volkns in di himlen / Shoyn toyznter yor mit mameshn tsar. / Di shlakht-felder vert farvaksn mit groz / Un fargisn mit blut zikh oyfsnay. // Vet di erd vern a shteyn, / Hart fun blut un zat fun geshray. / Oystrikenen veln di taykhn / Un di zilberne verbes iber zey! // Ven oyf shlofndike shtet faln bombes / Un derhargenen hayzer un mentshn, / Geyt ibern feld a shvangere froy / Un bet dem himl er zol mit a zun zi bentshn ».
} 
où battent les slogans et l'imagerie prototypique de la rhétorique soviétique, est radicalement différente de celle de ses premiers poèmes, et même de certaines autres pièces de Tsu loytere bregn consacrés à des thèmes moins ouvertement politiques. Alors que son écriture est habituellement épurée, laconique, suggestive, faisant la part belle à la déstructuration des strophes, des vers et des rimes, on se trouve ici face à une succession de quatrains relativement réguliers dans leur rythme et leur structure phonique, et dont les images, pour être encore allusives par endroits, laisse peu de doute sur la signification dont elles sont porteuses. Un tel ancrage dans un espace stable, à la fois idéologiquement, stylistiquement et géographiquement, relève cependant de l'exception : les lieux et les figures qui peuplent son œuvre (persona poétique incluse) sont le plus souvent suspendus entre l'ici et l'ailleurs, le présent et le révolu, l'air et l'eau, dressant des paysages discontinus dont les éléments surgissent et disparaissent sans jamais se souder au précédent. C'est qu'en réalité, le véritable territoire de Reïzl Zychlinsky n'est ni la Pologne, ni la Russie, ni New York ou la Californie - encore moins la France dont il n'est pour ainsi dire pas question dans son œuvre - mais l'espace poétique en tant que tel.

\section{Paysages intérieurs}

«La naissance d'un poème / Est comme la naissance d'une île ${ }^{18}$, écrit-elle dans Di november-zun [Le soleil de novembre]. Il surgit de mots et de sons qui, tels la lave d'un volcan durcissant après avoir jailli des eaux, fixent la coulée brûlante de la pensée, la condensent et la scellent dans la matière solide du langage poétique. L'image topographique n'est pas anodine : elle fait exister l'écriture comme lieu d'ancrage d'un Je poétique qui n'a d'autre lieu d'appartenance que ces roches insulaires formées de loin en loin, dans la rencontre de

\footnotetext{
${ }^{17}$ Karina von Tippelskirch, "Also das Alphabet vergessen?" Die jiddische Dichterin Rajzel Zychlinski, Marburg, Tektum Verlag, 2000. Dans le chapitre consacré au recueil Tsu loytere bregn, l'auteure constate un changement de style par rapport aux recueils précédents, notamment le premier, Lider, et insère ce glissement dans un phénomène plus vaste, identifié par David Roskies, selon lequel la Shoah marquerait le coup d'arrêt des expérimentations modernistes dans la poésie yiddish. Certains poèmes de Zychlinsky, cependant, mêlent des procédés stylistiques caractéristiques des deux périodes : c'est le cas, par exemple, de «Lider fun mayn heym » [Poèmes de mon foyer].

18 «Der geburt fun a lid / Iz vi der geburt fun an indzl». Cf. Reïzl Zychlinsky, « Dos lid », Di november-zun, Paris, [s.n.], 1977, p. 36.
} 
l'expérience et du signe, incandescences pétrifiées que la poétesse doit sans cesse façonner pour avoir un sol ferme sur lequel marcher.

Voilà qui nous invite à lire tout autrement les paysages ruraux, urbains ou cosmiques qui défilent dans l'œuvre de Reïzl Zychlinsky; à les lire, donc, non comme reflets de référents réels, comme mots renvoyant à la chose, mais comme lieux en soi, poursuivant sur le mode profane une tradition juive qui, depuis l'époque rabbinique, fait des textes sacrés l'objet principal sur lequel est censé se fixer le sentiment d'appartenance des communautés juives. Mais ces lieux, chez la poétesse, ne sont plus des points de convergence symboliques et éthiques, formateurs d'une culture collective ; ils sont l'expression d'une subjectivité qui est le seul véritable objet d'expression et qui, à l'instar des Inzikhistes - les poètes «introspectionnistes» américains favorisant la diction de l'expérience intérieure au détriment de la description naturaliste ou du message didactique, ne fait intervenir le monde extérieur qu'en tant que prolongement de la conscience d'un sujet. Ainsi des rues de New York devenues profondeurs sous-marines, où le métal et le marbre prennent vie, acquièrent une dimension quasi organique :

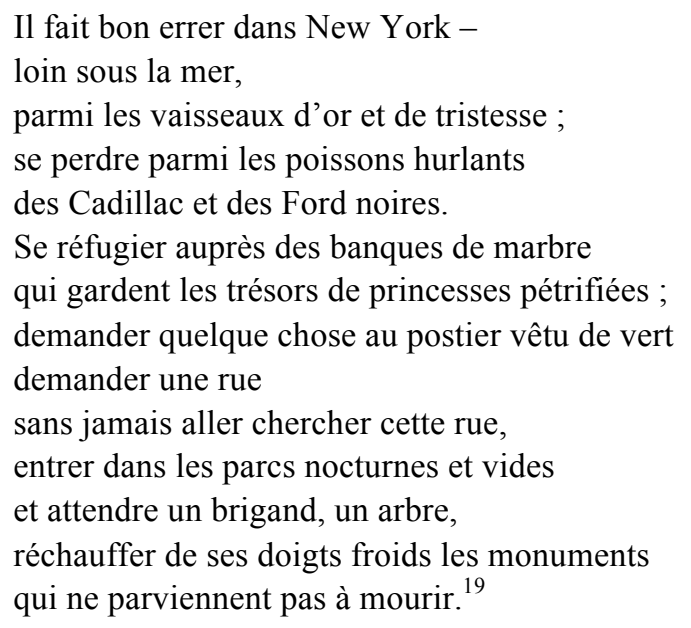

${ }^{19}$ Portes muettes, op. cit., p. 21. Reïzl Zychlinsky, « Nyu-York », Shvaygndike tirn, New York, Yidish PEN-klub, 1962, p. 8: «S'iz gut tsu vandern in Nyu-York - / Tif untern yam, / Tsvishn shifn mit gold un mit troyer; / Plontern zikh tsvishn revendike fish - / Di shvartse kadilaks un fords. / Rateven zikh nebn marmorene benk, / Vos hitn oytsres fun farshteynerte printsesins ; / Fregn epes bay a grinem briv-treger, / Fregn oyf a gas / Un gornisht geyn zukhn di gas, / Arayngeyn in leydike un nakhtike parkn / Un vartn oyf a royber, oyf a boym, / Onvaremen mit a finger kalte monumentn / Vos kenen nisht shtarbn. » En réalité, dans l'avant-dernier vers, le texte original dit « réchauffer du doigt les froids monuments ». 
On ne peut s'empêcher de voir, dans cette errance surréaliste à travers la métropole dépeuplée, une plongée en soi-même : la ville qui ne dort jamais semble paradoxalement vide, engourdie, et immergée dans le temps ralenti du rêve. Le quadrillage urbain répond à une cartographie mentale où se mêlent le vacarme et le silence, le perceptible et l'imaginaire, les machines urbaines et la faune marine, le froid du marbre et la chaleur de la peau, la fulgurance moderne et le non-temps du conte - toutes les contradictions d'un soi qui n'est plus séparé du monde extérieur, mais se confond avec les éléments qui l'entourent, vivants ou non, s'absorbe en eux jusqu'à devenir paysage. C'est peut-être cela qui, plus que toute autre chose, caractérise la poésie de Reïzl Zychlinsky : une porosité de l'être qui, faute de frontières fermes entre les objets et les temporalités, se trouve sans cesse traversé et incorporé par des réalités autres, au point de devenir lui-même vent, arbre, neige, mer ou fleur.

Et si les poèmes sont des «îles », c'est que chacun d'entre eux rassemble dans une forme stable une incarnation passagère de ce corps dont le sentiment de soi est à la fois partout et nulle part. L'île, donc, comme terre conquise contre la dissolution de soi, extirpée du fond des eaux troubles dans lesquelles viennent s'engloutir des pans entiers de l'existence. Telle la conscience éclatée de la persona poétique, livrée à une errance qui, chaque fois, naît de la disparition des lieux de reconnaissance passés, les poèmes apparaissent comme autant de masses flottantes, dont les liens, profonds et sous-marins, demeurent invisibles à qui n'arpente que leur surface. D'où des images qui, elles aussi, fonctionnent par irruptions soudaines de couleurs et de formes, exposant le lecteur à des représentations aussi cinglantes que déroutantes, dont la charge picturale flirte tantôt avec le surréalisme, tantôt avec l'expressionisme. Au premier, la poétesse emprunte l'étrangeté de ses représentations, et en particulier des représentations du corps humain, source de fascination en même temps que de malaise: les mains qui grandissent au point d'envahir toute la table, les os de l'enfant qu'on « entend durcir » ${ }^{20}$ à l'automne, la tête aux grands «yeux juifs » ${ }^{21}$ émergeant de la terre, «surgeon maudit qui ne peut mourir » sont autant d'images qui démembrent l'unité du corps, le pétrifient ou le désarment, livrant la représentation du soi à une déliquescence qui rappelle tantôt la

\footnotetext{
${ }^{20}$ " Un ikh her vi es vern hart / Di vaykhe beyndlekh fun mayn kind.» Cf. Reïzl Zychlinsky, « Harbst » [Automne], Tsu loytere bregn, op. cit., p. 14.

${ }^{21} \mathrm{Ibid}$., p. 5 : voir le poème intitulé « Mayne yidishe oygn » [Mes yeux juifs]: « Nor mayn kop shtekt fun keyver aroys, / A farsholtn geviks, vos kon nisht shtarbn. ».
} 
« construction molle» d'un Dali tantôt la mémoire sanglante et statufiée d'un Magritte. Au second, Reïzl Zychlinsky doit le tranchant de ses descriptions et l'explosion de ses teintes, parfois crues, toujours franches, qui rappelle le goût des avant-gardes artistiques de l'entre-deux-guerres pour les couleurs primaires. Que l'on pense au «chat vert» qui revient dans bien des poèmes, évocateur des chevaux bleus de Franz Marc, à la 《 robe rouge $»^{22}$ que revêt la persona pour guetter la mort et surtout à la « cruche bleue» dont la teinte s'accorde au regard maternel. Dans "Shneyen» [Neiges], le silence floconneux des grandes étendues enneigées, avant d'être zébré de feu et de sang par la guerre, est réveillé par un souvenir bleu : «Dans le tourbillon je vois mon shtetl, ma maison, / [...] / Le bleu que j'ai tété à la mamelle. Je cherche ma mère, ses yeux bleus $»^{23}$. Ailleurs, dans «Vayt, vayt iz dos shtetl» [Loin, loin est le shtetl], le sujet poétique voit à nouveau ressurgir le bleu du souvenir « sous la neige épaisse et coite ${ }^{24}$ dans laquelle il est enseveli :

Ma mère attendait d'heure en heure

L'eau qui ne venait pas -

Mais la rivière argentée miroitait, miroitait

Et la cruche était bleue. ${ }^{25}$

Et enfin, dans « Lider fun mayn heym » [Poèmes de mon foyer] :

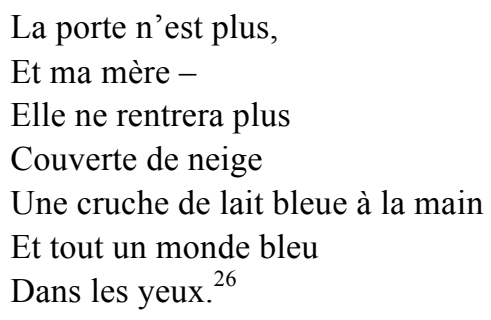

Le rapprochement répété du bleu avec le souvenir de la mère et du shtetl crée chez le lecteur une sorte de réflexe associatif, qui le connecte à

\footnotetext{
22 «Quand les fenêtres », dans Portes muettes, op. cit., p. 46.

23 «In gevirbl ze ikh mayn shtetl, mayn heym, / [...] / Di bloykeyt vos kh'hob mit der milekh gezoygn. / Di mame zukh ikh, ire bloye oygn. » Reïzl Zychlinsky, « Shneyen », Tsu loytere bregn, op. cit., p. 40.

${ }^{24}$ Ibid., p. 15 : « unter shneyen gedikhte un shtile».

${ }^{25}$ Ibid., «Flegt vartn di mame oyf dem vaser, / A sho un nokh a sho - / Nor der taykh hot gezilbert, gezilbert, / Un der krug iz geven blo ».

${ }^{26}$ Ibid., p. 16 : «Di tir iz nishto, / Un di mame - / Zi vet mer nisht aheymkumen / A farshneyte / Mit a bloyen krug milkh in der hant / Un mit a bloyer velt / In di oygn ».
} 
la symbolique personnelle habitant l'univers de la poétesse. Pour peu que l'on considère l'œuvre dans son ensemble, et non pas chaque poème comme une unité isolée, la mention de la couleur bleue suffit alors à évoquer les souvenirs qui lui sont associés, sans qu'il soit toujours nécessaire de les mentionner explicitement. C'est cette présence suggérée qui parcourt la poésie de Reïzl Zychlinsky, bien souvent laconique mais riche des possibilités qui s'ouvrent en elle, rendant le lecteur partie prenante des images et du sens que produit chaque poème.

Ses paysages, loin d'être purement intérieurs, abolissent les frontières entre intériorité et extériorité, connectant l'autre à ses représentations intimes et faisant de la nature une entité animée, douée de vie propre, dans laquelle se prolonge la conscience humaine. Plus que l'expérience spécifique d'une réalité naturelle ancrée dans un temps et un lieu précis, c'est d'ailleurs une vision symboliste de l'espace qui prédomine, rivée à quelques éléments archétypaux caractéristiques du conte ou du mythe : la mer, la forêt, la montagne, le désert, milieux à la fois topographiques et métaphoriques, contenus en même temps que contenants de l'être, apparaissent comme les garants d'une pérennité sur laquelle, après la Seconde Guerre mondiale, vient se greffer la mémoire du disparu. En effet, si les poèmes de Tsu loytere bregn sont souvent datés et situés, au point d'exacerber leur lien à l'Histoire, et si l'on trouve dans les recueils plus tardifs des références explicites au génocide ${ }^{27}$, la dimension mémorielle acquiert également une dimension plus cosmique, qui transcende l'anéantissement de Gombin et de tous les shtetlekh du même type, dépasse la fixation sur des lieux « témoins » rigoureusement choisis pour établir une sorte de parenté entre le monde intérieur de la poétesse - troué par la disparition de ses lieux et de ses proches - et le monde cosmique - frappé d'une étrangeté qui ne cesse de figurer cette même absence. La mort violente de la mère, exterminée à Treblinka avec les frères et sœur de Zychlinsky alors que cette dernière avait réussi à fuir en Union soviétique, ne prend pas toujours la forme d'un événement; elle peut aussi se donner comme absence inhérente, dans laquelle s'enracine la volonté de la poétesse de créer une nouvelle filiation avec le cosmos :

\footnotetext{
${ }^{27}$ On peut citer, entre autres exemples, le poème de Di november-zun intitulé « S'ken zayn » [Il se peut], qui commence sur ces mots : "S'ken zayn / Ikh hob haynt gezen dokter Mengele / Trinken a gloz tey / In Tel-Aviv / Baym yam - » [Il se peut / Que j'aie vu aujourd'hui le docteur Mengele / Boire un verre de thé / À Tel-Aviv / Au bord de la mer - ». Cf. Di november zun, op. cit., p. 73.
} 


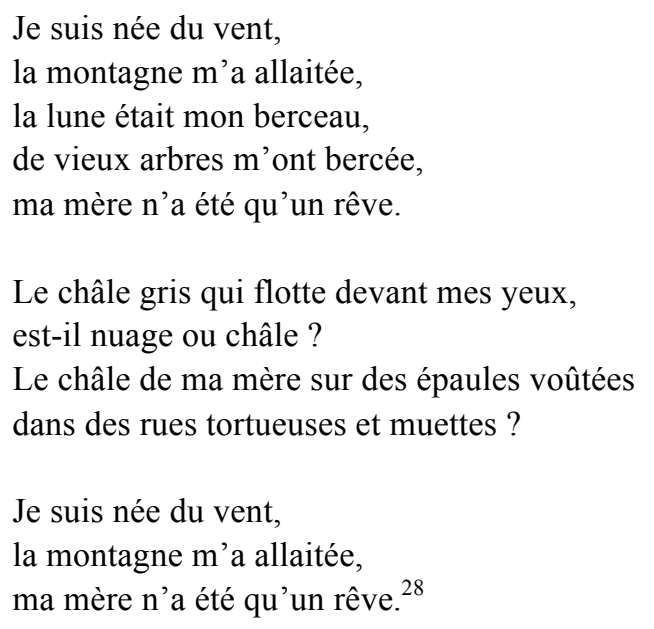

L'absence de la mère, remplacée par une nature qui, parce qu'elle est à la fois nulle part et partout, enveloppe le sujet poétique dans son non-corps, apparaît comme la forme exacerbée d'une spectralité qui traverse l'ensemble de l'œuvre. Rien n'a de contours définis: ni les lieux, ni les choses, ni même le «je » qui s'exprime ; tout est toujours noyé dans un magma de formes dont on ne sait où elles commencent, pas plus qu'on ne sait où elles s'achèvent. Il en est de même des êtres humains, qui sont obstinément absents du monde poétique tout en saturant chacun des vers qui ne les mentionne pas. Car si les êtres ne sont pas là, le monde inanimé semble comme habité de leur non-existence : anthropomorphisé tout entier, il étend jusqu'à l'extrême la communauté à laquelle appartient la poétesse, qui ne se limite plus ni au groupe juif, ni à l'espèce humaine, ni même au monde animal, mais englobe tout ce avec quoi elle entre en contact physique ou mental. C'est à la terre que s'adresse « Tfile » [Prière] ${ }^{29}$, relais d'un interlocuteur divin qui n'est plus là pour façonner la glaise à son image ; et c'est aux collines, au fleuve, à l'arbre, au nuage, que la poétesse se compare et dit son amour ${ }^{30}$, dans une sorte de symbiose avec le cosmos qui transcende la séparation brutale et

\footnotetext{
${ }^{28}$ Portes muettes, op. cit., p. 79. Reïzl Zychlinsky, « Geboyrn hot mikh der vint » [Je suis née du vent], Shvaygndike tirn, op. cit., p. 53 : «Geboyrn hot mikh der vint, / Gezoygn der barg, / Di levone iz geven mayn vigl, / Alte beymer hobn gevigt mikh tsum shlof, / Di mame iz geven a kholem. // Dos groye tukh vos flatert far di oygn, - / Iz es a volkn oder a fatsheyle ? / Der mames fatsheyle oyf geboygener pleytse / In geslekh krume un shtile ? // Geboyrn hot mikh der vint, / Gezoygn der barg, / Di mame iz geven a kholem ».

${ }^{29}$ Shvaygndike tirn, op. cit., p. 111 et Portes muettes, op. cit., p. 147.

${ }^{30}$ « Kh'hob lib di berglekh», Shvaygndike tirn, op. cit., p. 154 et « J'aime les collines », Portes muettes, op. cit., p. 203.
} 
définitive d'avec le foyer natal, assurant une sorte d'involution vers le giron d'une nature dont l'existence se compte en éternités.

Plusieurs fois comparée à la poésie japonaise ${ }^{31}$ pour sa propension à saisir, dans quelques mots choisis, l'essence d'une rencontre inattendue entre l'âme et le monde, la sensibilité de Reïzl Zychlinsky peut sembler aux antipodes d'une tradition juive qui met l'homme, image du divin, au centre de toute chose et n'admet de relation à la nature que culturellement codée. En témoigne ce poème qui prend le contre-pied du récit de la Genèse et dans lequel le corps de la mère, noyé par la pluie, redevient glaise ${ }^{32}$, abolissant toute mise en forme symbolique de la confusion primordiale. Ici, c'est la terre qui est le centre et l'aboutissement de toute chose ; la nature, défaite des filtres culturels qui en familiarise la perception, devient un principe désorganisateur dans lequel se répercute la dissolution identitaire du moderne, effaçant les contours qui permettaient de diviser le monde en catégories et en entités distinctes. Elle prend forme humaine, certes, dans la reprise de certains topo $\ddot{~ d e ~ l a ~ l i t t e ́ r a t u r e ~ d e ~ l a ~ c a t a s t r o p h e ~ q u i ~ a c c u s e n t ~ s o n ~ s i l e n c e ~ e t ~ s o n ~}$ indifférence au meurtre, mais elle accède surtout à une inquiétante étrangeté née de la troublante confusion des corps animés et inanimés. Cette confrontation nue à un monde que l'héritage culturel ne suffit plus à faire signifier, Reïzl Zychlinsky la coule dans des formes poétiques qui reconfigurent le rapport de l'homme au monde, et apparaissent donc comme une manière de réélaborer du sens dans une tentative toujours renouvelée de nommer «cette chose qui flotte sur 1'Hudson " ${ }^{33}$, image d'un soi sans contours que peuplent les morts et qui ne parvient à se constituer en «îles » que dans le pouvoir de symbolisation du langage créateur.

\footnotetext{
${ }^{31}$ On trouve déjà une comparaison de ce type dans la préface d'Itzik Manger à Lider, réitérée par Rachel Ertel dans son introduction à Portes muettes.

${ }^{32}$ Reïzl Zychlinsky, « Der regn zingt », Der regn zingt, Varsovie, Biblyotek fun yidish PEN-klub, 1939, p. 14.

${ }^{33}$ Reïzl Zychlisnky, « Vos shvimt dort oyfn Hodson? », Shvaygndike tirn, op. cit., p. 7 et « Quelle est cette chose qui flotte sur l'Hudson ?», Portes muettes, op. cit., p. 20.
} 